\title{
Axis inhibition protein 2 polymorphisms may be a risk factor for families with isolated oligodontia
}

\author{
HAN QIN $^{1}$ and JUN CAI ${ }^{2}$ \\ ${ }^{1}$ Department of Stomatology, The First People's Hospital of Lianyungang City, Lianyungang, Jiangsu 222002; \\ ${ }^{2}$ Department of Anesthesia, The Third People's Hospital of Lianyungang City, Lianyungang, Jiangsu 222006, P.R. China
}

Received February 4, 2014; Accepted October 20, 2014

DOI: $10.3892 / \mathrm{mmr} .2014 .2900$

\begin{abstract}
The objective of the present study was to search for Msh homeobox 1 (MSX1), paired box gene 9 (PAX9), ectodysplasin-A (EDA) and axis inhibition protein 2 (AXIN2) variants in a family with isolated oligodontia and analyse the pathogenesis of mutations that result in oligodontia phenotypes. Members of a single family (but of different descent) with oligodontia and unrelated healthy controls were enrolled in our study. Genomic DNA was isolated from blood samples. Mutation analysis was performed by amplifying MSX1, $P A X 9$, $E D A$ and $A X I N 2$ exons as well as their exon-intron boundaries and sequencing the products. DNA sequencing of the AXIN2 gene revealed three mutations in the two patients with oligodontia: a homozygotic silent mutation c.1365A $>\mathrm{G}$ (p.Pro455=) in exon 3, two c. $956+16 \mathrm{~A}>\mathrm{G}$ mutations (II-1: homozygosis; III-1: heterozygosis) and c. $1200+71 \mathrm{~A}>\mathrm{G}$ (homozygosis) in the intron, which possibly contributed to structural and functional changes in proteins. The heterozygotic mutations c.1365A $>\mathrm{G}$ and c. $1200+71 \mathrm{~A}>\mathrm{G}$ were identified in the proband's mother (II-2). No mutations were detected in the MSX1, PAX9 and $E D A$ genes of oligodontia patients. The findings suggest that the c. $956+16 \mathrm{~A}>\mathrm{G}, \mathrm{c} .1365 \mathrm{~A}>\mathrm{G}$ and c. $1200+71 \mathrm{~A}>\mathrm{G}$ mutations of AXIN2 may be responsible for the oligodontia phenotype in this family, but these findings require further study.
\end{abstract}

\section{Introduction}

Abnormalities in tooth number affect approximately $20 \%$ of the population (1). Oligodontia is defined as the congenital absence of six or more permanent teeth, excluding the third molar. Tooth loss may appear either as a feature of multi-organ syndromes or as a non-syndromic isolated trait. Individuals

Correspondence to: Dr Han Qin, Department of Stomatology, The First People's Hospital of Lianyungang City, 182 Tongguan Road, Lianyungang, Jiangsu 222002, P.R. China

E-mail: qinhan2005@163.com

Key words: oligodontia, axis inhibition protein 2, gene mutation, gene polymorphism with missing teeth have numerous problems with aesthetics, phonetics and mastication. The development of dentition is a notable process that encompasses a complex series of epithelial-mesenchymal interactions involving growth factors, transcription factors, signal receptors and other soluble morphogens (2). Any disturbance in this tightly balanced process may result in tooth agenesis or other dental defects. Numerous genes that underlie dental defects have been identified; however, the occurrence of non-syndromic cases remains poorly understood. Previous studies have demonstrated that Msh homeobox 1 (MSX1), paired box gene 9 (PAX9), ectodysplasin-A (EDA) and axis inhibition protein 2 (AXIN2) are key regulators of tooth development. Lammi et al suggested that oligodontia may be caused by mutations of the AXIN2 gene (3), which is localised on chromosome 17q21-q25. AXIN2 is known as an intracellular antagonist of Wnt signalling that is expressed in the dental mesenchyme, odontoblasts and enamel knots (4). In humans, mutations in AXIN2 cause tooth agenesis affecting permanent teeth, predominantly including permanent molars, lower incisors and upper lateral incisors $(5,6)$. The objective of the present study was to identify the AXIN2 mutations responsible for oligodontia in a family with non-syndromic oligodontia. In addition, we attempted to explore genotype-phenotype correlations that could improve our current understanding of different mutations based on the pattern of tooth agenesis.

\section{Materials and methods}

Patients and controls. The female proband was a patient of the Department of Stomatology at the First People's Hospital of Lianyungang City, Lianyungang, China. A pedigree of this family was constructed by extended interviews. In this study, all individuals presented with normal physical development and normal intelligence, and the clinical examination for other ectodermal abnormalities of the nails, hair, skin and sweat glands did not reveal any defects. Thus, we suggested that the family exhibited non-syndromic oligodontia. Retrospective data were reviewed and the diagnosis of oligodontia was verified by panoramic dental radiographs for all available family members. Eight members of the family were studied, with three members being affected, one of which was deceased, and five unaffected. Furthermore, 60 unrelated individuals (of different ages and 
Table I. MSXI primer sequences.

\begin{tabular}{llllc}
\hline Region & \multicolumn{1}{c}{$\begin{array}{c}\text { Forward primer } \\
\left(5^{\prime}-3^{\prime}\right)\end{array}$} & \multicolumn{1}{c}{$\begin{array}{c}\text { Reverse primer } \\
\left(5^{\prime}-3^{\prime}\right)\end{array}$} & $\begin{array}{c}\text { Size } \\
(\mathrm{bp})\end{array}$ & $\begin{array}{c}\text { Annealing } \\
\text { temperature }\left({ }^{\circ} \mathrm{C}\right)\end{array}$ \\
\hline Exon 1 & GGCTGCTGACATGACTTCTTTGC & TTGGAACCTTCTTCCTGGGTG & 642 & 65 \\
Exon 2 & CCAGAAGCAGTACCTGTCCAT & TCAGGGATCAGACTTCGGAGA & 506 & 65 \\
\hline
\end{tabular}

Table II. PAX9 primer sequences.

\begin{tabular}{lllcc}
\hline Region & \multicolumn{1}{c}{$\begin{array}{c}\text { Forward } \\
\text { primer }\left(5^{\prime}-3^{\prime}\right)\end{array}$} & \multicolumn{1}{c}{$\begin{array}{c}\text { Reverse primer } \\
\left(5^{\prime}-3^{\prime}\right)\end{array}$} & $\begin{array}{c}\text { Size } \\
(\mathrm{bp})\end{array}$ & $\begin{array}{c}\text { Annealing } \\
\text { temperature }\left({ }^{\circ} \mathrm{C}\right)\end{array}$ \\
\hline Exon 1 & TTTCATCGGGGCACAGACTTCC & TCCAGTAGCCAGCGTTCACA & 391 & 65 \\
Exon 2A & TTCCGTTCGGCTATGTTCAGG & CGAGTAGATGTGGTTGTAGGG & 645 & 65 \\
Exon 2B & GATACAACGAGACGGGCTCGAT & TATGTGGCAAGGAAACCAGGGG & 655 & 65 \\
Exon 3 & TTATAAGCCCTCCAGCTCTCCGT & GTGTTCCATCGCCTAAATCCC & 304 & 65 \\
Exon 4 & GAGCATTGCTGGCTTACTCAGA & TTTCAAGGCAGAAGGGTTGGAGG & 477 & 65 \\
\hline
\end{tabular}

Table III. EDA primer sequences.

\begin{tabular}{lllll}
\hline Region & \multicolumn{1}{c}{$\begin{array}{c}\text { Forward } \\
\text { primer }\left(5^{\prime}-3^{\prime}\right)\end{array}$} & \multicolumn{1}{c}{$\begin{array}{c}\text { Reverse } \\
\text { primer }\end{array}$} & $\begin{array}{c}\text { Size } \\
(\text { bp })\end{array}$ & $\begin{array}{c}\text { Annealing } \\
\text { temperature }\left({ }^{\circ} \mathrm{C}\right)\end{array}$ \\
\hline Exon 1 & TATTGCCGCCTGTCAGAGGTC & TGGGAGCGTCGCCAACTTTT & 762 & 65 \\
Exon 2 & ATGGGCTCAGGCTTTAGACA & TGCCCTACCAAGAAGGTAGT & 318 & 65 \\
Exon 3 & GGCTGGGGAGGTTTGATAGTTG & GAAAGAAGGGCAGGGAGAAG & 423 & 65 \\
Exon 4 & ACCCGAAGTCAGGAGTTTGAGA & CATCCCTTATTGCACACCAACC & 555 & 65 \\
Exon 5 & TGTCAGGGCAGGAAACAGAA & TTTGATCCTGGCAAGACACC & 435 & 65 \\
Exon 6 & GCAGTAACATCCCAAGACAG & TATGCTCAGGACAGCCAGTA & 360 & 65 \\
Exon 7 & ATACCCTGGTTGCACTGGGATAG & GCCCAAAGCAGGAAGTTAGCCATT & 362 & 65 \\
Exon 8 & CAATGCCTGTCACCTGTCCTTTC & ACCTCACTCCACAGCAGCACTTA & 520 & 65 \\
\hline
\end{tabular}

genders) who were not affected with tooth agenesis (excluding third molars) were used as controls. The study was approved by the Institutional Review Board as well as the Ethics Committee of the First People's Hospital of Lianyungang City.

DNA extraction and polymerase chain reaction (PCR) of candidate genes. Peripheral blood samples from all members of the family and controls were collected from the Department of Stomatology at the First People's Hospital of Lianyungang City. Genomic DNA was extracted from these samples with the QIAamp DNA blood mini kit (Qiagen, New York City, NY, USA). To screen for putative mutations, two exons of MSX1 (GenBank accession number M97676), four exons of PAX9 (GenBank accession number AJ238381), ten exons of AXIN2 (GenBank accession number AE006463) and eight exons of EDA (GenBank accession number U59228), as well as their exon-intron boundaries, were amplified by PCR with the use of primers that were previously reported (7) or designed using the Primer 3 online application (http://frodo. wi.mit.edu/primer3/input.htm). The primer sequences and optimal annealing temperature for each primer pair are shown in Tables I-IV. The PCR amplifications used the GC-rich PCR system (Roche, Nutley, NJ, USA) and the PrimeSTAR PCR system (Takara Bio, Inc., Otsu, Japan). The amplified fragments of AXIN2 from individuals of the affected family and controls were gel-purified with the MinElute gel extraction kit (Qiagen) according to the manufacturer's instructions. The sequencing analyses were performed with an ABI BigDye ${ }^{\mathrm{TM}}$ terminator cycle sequencing ready reaction kit with AmpliTaq DNA polymerase on an ABI PRISM ${ }^{\circledR} 377$ XL DNA sequencer (Applied Biosystems Life Technologies, Carlsbad, CA, USA). Finally, the mutations and polymorphisms in AXIN2 at the genomic and protein levels were analysed using MegAlign 5.01 software (DNASTAR, Inc., Madison, WI, USA), Polyphen-2 software (Harvard University, Cambridge, MA, USA) and exonic splicing enhancer (ESE) Finder 3.0 software (Cold Spring Harbor Laboratory, Cold Spring Harbor, NY, USA).

\section{Results}

Clinical examination. We studied a family in which oligodontia was segregating in an autosomal-dominant manner. 
Table IV. AXIN2 primer sequences.

\begin{tabular}{|c|c|c|c|c|}
\hline Region & $\begin{array}{c}\text { Forward } \\
\text { primer }\left(5^{\prime}-3^{\prime}\right)\end{array}$ & $\begin{array}{l}\text { Reverse } \\
\text { primer }\end{array}$ & $\begin{array}{l}\text { Size } \\
\text { (bp) }\end{array}$ & $\begin{array}{c}\text { Annealing } \\
\text { temperature }\left({ }^{\circ} \mathrm{C}\right)\end{array}$ \\
\hline Exon 2 & AGAGAGACCACGCCGATTG & CATGGCCAGCAGTCCTAAC & 1017 & 64 \\
\hline Exon 3 & CTTTCTGCCCAGGTGAGC & CCCACCAAACTGATGTCC & 424 & 61 \\
\hline Exon 4 & AAGCCAAGTTTCCATAGATGA & GTTCTTTTCTCTCССTTTGCC & 471 & 62 \\
\hline Exon 5 & CGCCTTGCCATCCACTC & GCACATGCGCACACAGC & 443 & 64 \\
\hline Exon 6 & ATGGGTTGCGTGTGGGT & GGGCTGGTGACACGAAAG & 883 & 63 \\
\hline Exon 7 & CGCATTACAGGCATTTAGTT & AAGATGGCAGGAGCAAATAG & 499 & 62 \\
\hline Exon 8 & GACTATTTGCTCCTGCCATC & GACTTGCCTCACAGATCCTG & 674 & 62 \\
\hline Exon 9 & CAGGGTCTTGGTTGGGTCT & CAGGACATGGATGGCAACA & 408 & 64 \\
\hline Exon 10 & CATGTGGGGTTGGACTGTG & CGGCTGCTGCTTCGTTATT & 466 & 66 \\
\hline Exon 11 & AGTCCCAGCTGCCGTCTTA & CCACTGGCCGATTCTTCC & 431 & 65 \\
\hline
\end{tabular}

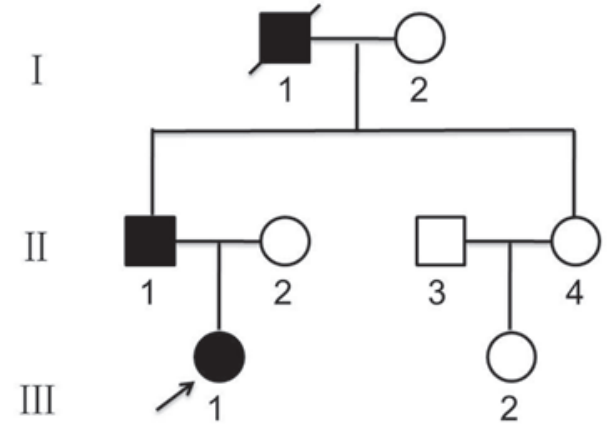

Figure 1. Pedigree of the family.

A pedigree of this family was constructed by extended interviews (Fig. 1). Retrospective data were reviewed, and the diagnosis of oligodontia was verified based on panoramic dental radiographs of all available family members. Clinical and radiographic examinations revealed that the proband's father (II-1) was missing 12 permanent teeth: one permanent maxillary lateral incisor, two permanent maxillary canines, three permanent maxillary premolars, two permanent mandibular central incisors, two permanent mandibular lateral incisors and two permanent mandibular premolars (Fig. 2). The proband (III-1) was missing 12 permanent teeth: two permanent maxillary lateral incisors, two permanent maxillary canines, four permanent maxillary premolars and four permanent mandibular premolars (Fig. 3).

Mutation analysis. DNA sequencing of the AXIN2 gene revealed three mutations in the two patients with oligodontia: a homozygotic silent mutation c.1365A $>\mathrm{G}$ (p.Pro455=) in exon 3, two mutations c. $956+16 \mathrm{~A}>\mathrm{G}$ (II-1: homozygosis; III-1: heterozygosis) and c. $1200+71 \mathrm{~A}>\mathrm{G}$ (homozygosis) in the intron. In addition, the heterozygotic mutations c.1365A $>\mathrm{G}$ and c. $1200+71 \mathrm{~A}>\mathrm{G}$ were identified in the proband's mother (II-2; Fig. 4). The three mutations were thought to be known polymorphisms (rs9915936, rs35285779 and rs8078753) according to a bioinformatics analysis. Polyphen-2 software was used to predict the possible impact of the amino acid substitution on the structure and function of the AXIN2

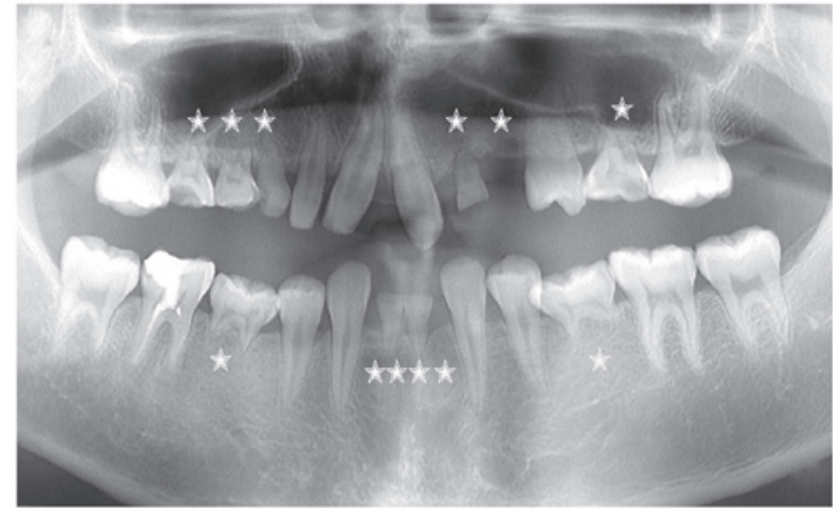

Figure 2. Panoramic radiograph showing that the proband's father (II-1) was missing 12 permanent teeth: one permanent maxillary lateral incisor, two permanent maxillary canines, three permanent maxillary premolars, two permanent mandibular central incisors, two permanent mandibular lateral incisors and two permanent mandibular premolars.

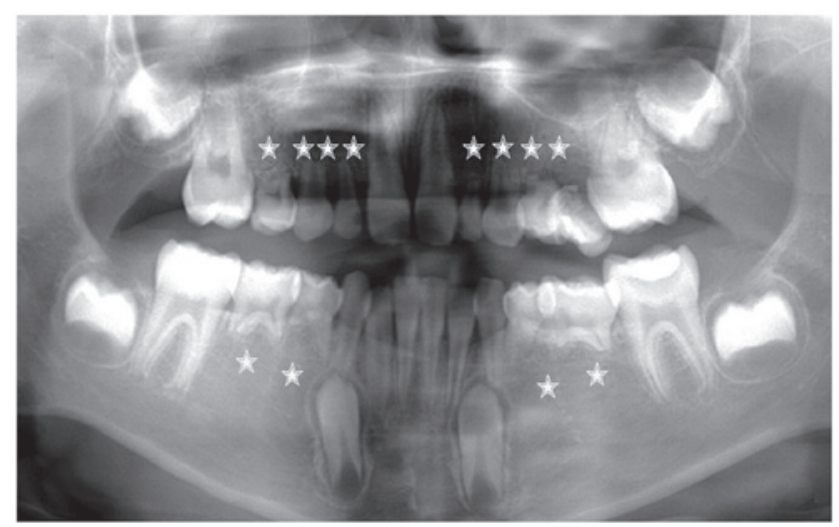

Figure 3. Panoramic radiograph showing that the proband (III-1) was missing 12 permanent teeth: two permanent maxillary lateral incisors, two permanent maxillary canines, four permanent maxillary premolars and four permanent mandibular premolars.

protein. The change was predicted to be benign. We also used ESE Finder 3.0 software to predict whether the change would alter exonic splicing enhancers. The $\mathrm{A}$ to $\mathrm{G}$ nucleotide change did not affect any predicted exonic splicing enhancer site. No 


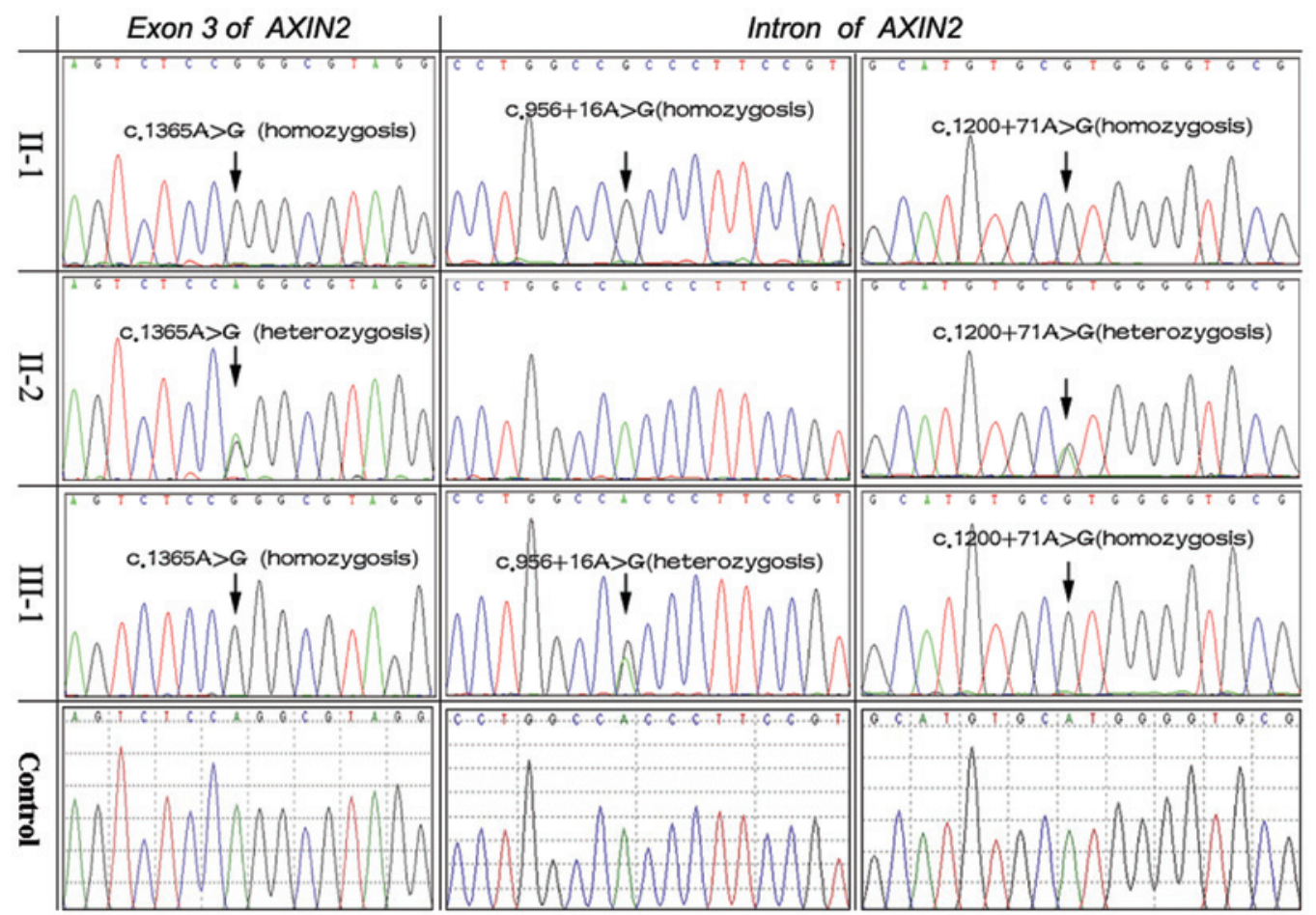

Figure 4. DNA sequencing of the axis inhibition protein 2 gene reveals three mutations in the two patients with oligodontia: a homozygotic silent mutation c.1365A > G (p.Pro455=) in exon 3, two c.956+16A>G mutations (II-1: homozygosis; III-1: heterozygosis) and c.1200+71A>G (homozygosis) in the intron. The heterozygotic mutations c.1365A $>\mathrm{G}$ and c.1200+71A $>\mathrm{G}$ were identified in the proband's mother (II-2).

mutations were detected in EDA, $P A X 9$ or $M S X 1$. In addition, the family members of other descent and the unrelated healthy controls had no mutations in EDA, MSX1, PAX9 or AXIN2.

\section{Discussion}

Oligodontia appears as a feature of multi-organ syndromes as well as a non-syndromic isolated character. We analysed a family in which oligodontia was segregating in an autosomal-dominant manner in order to define the clinical features of oligodontia and to localise the gene locus behind this anomaly. Our clinical examinations and interviews of the kindred revealed that the affected members were missing more than six teeth but had no other systemic abnormality. These individuals were diagnosed with non-syndromic oligodontia.

Numerous genes underlying dental defects have been identified (8-10); however, the occurrence of non-syndromic oligodontia remains poorly understood. Based on results from familial studies, Lammi et al suggested that oligodontia may be caused by mutations of the AXIN2 gene (3). The protein product of $A X I N 2$ is a negative regulator of the canonical Wnt pathway and suppresses signal transduction by promoting the degradation of $\beta$-catenin $(11,12)$. The inactivating mutations described in AXIN2 that lead to oligodontia and higher susceptibility to colon cancer affected incisor development in 11 out of 12 cases. Mostowska et al suggested that AXIN2 polymorphic variants may be associated with both hypodontia and oligodontia (13). This study from Poland suggested that two AXIN2 variants were in strong linkage disequilibrium with each other: the silent mutation c. $2062 \mathrm{C}>\mathrm{T}$ and the intronic variant c. $956+16 \mathrm{~A}>\mathrm{G}$. Linkage disequilibrium refers to the non-random association of linked genes. This is the tendency of the alleles of two separate but already linked loci to be found together more frequently than usual. c. $2062 \mathrm{C}>\mathrm{T}$ was predicted to disrupt exonic splicing enhancer sequences, and although a synonymous change, it could contribute to the tooth agenesis phenotype. The c.956+16A $>\mathrm{G}$ mutation was also suggested to exert a possible effect on splicing as it creates an additional donor-splicing site within the sequence of exon 2. In our study, three AXIN2 variations (including the c $.956+16 \mathrm{~A}>\mathrm{G}$ mutation) were identified in the proband and her father, which confirm the significance of $A X I N 2$ in tooth development.

Previous studies have suggested that different tooth types are regulated by independent gene expression $(14,15)$. Numerous Wnt genes are expressed in developing teeth, and changes in their expression may be one of the factors determining tooth agenesis (16). There is evidence that genes associated with the Wnt pathway are differentially expressed in molars as opposed to incisors. Studies in mice targeting lymphoid enhancer-binding factor 1 (LEF1) function, a transcription factor that can be activated by Wnt proteins, revealed that transgene molar expression of the LEF1 promoter during molar development was observed only in E12.5 embryos and was absent in E13.5 embryos. Conversely, LEF1 promoter expression during incisor tooth development persisted from E12.5 to E17.5 (17). It is reasonable to hypothesise that AXIN2 alleles affect incisors more often due to the persistent expression of LEF1 in these developing teeth compared with molars. In the family studied, II-1 was missing one permanent maxillary lateral incisor, two permanent maxillary canines, three permanent maxillary premolars, two permanent mandibular central incisors, two permanent mandibular lateral incisors and two permanent mandibular premolars, while III-1 was missing two permanent maxillary lateral incisors, 
two permanent maxillary canines, four permanent maxillary premolars and four permanent mandibular premolars. Neither of the patients had missing molars (with the exception of the third molars). These results may therefore suggest that multiple AXIN2 variants could contribute to sporadic forms of common incisor agenesis in humans, which is in agreement with previous studies on the frequency of tooth loss with AXIN2 mutations (13).

The same type of mutation (c.1365A $>\mathrm{G}$ and c.1200+71A $>\mathrm{G}$ ) but in heterozygotic form was identified in the proband's mother. Possible reasons for this may be gene polymorphism, multiple gene factors and environmental factors. First, the rationale for performing the joint effect analysis of the studied single nucleotide polymorphism (SNP) is that, under a mixture of various pollutants, the contribution of polymorphisms in various metabolising enzymes could exert an additive effect in disease susceptibility that is only possible to identify when considering the SNPs in combination. This analysis revealed that the combination of the c. $956+16 \mathrm{~A}>\mathrm{G}, \mathrm{c} .1365 \mathrm{~A}>\mathrm{G}$ and c. $1200+71 \mathrm{~A}>\mathrm{G}$ homozygotic mutations may increase the oligodontia risk compared with heterozygotic mutations and polymorphisms. The identification of the causative genetic variants involved in the phenotypes of interest remain a difficult task. In light of the non-independence of mammalian dental traits (18), adequate samples of various pedigrees and references to established databases are required to determine the association of the genotype and phenotype in oligodontia. Although the study subjects come from the same geographical area, notable aspects concerning the source of cases and controls have to be taken into account. Our findings may imply that c.956+16A>G (rs35285779, homozygosis), c.1365A >G (p.Pro455=, rs9915936, homozygosis) and c.1200+71A $>\mathrm{G}$ (rs8078753, homozygosis) mutations are risk factors for oligodontia in the Chinese population. However, due to the small sample size and the limited number of studies, our results should be interpreted with caution.

The second explanation could be the multiple gene factors. The development of dentition is a notable process that encompasses a complex series of epithelial-mesenchymal interactions involving growth factors, transcription factors, signal receptors and other soluble morphogens. To date, over 300 genes have been identified as being involved in tooth development (19). This fact highlights the significance of other presently unknown genes and developmental factors in tooth development and in the etiology of dental anomalies. Tooth agenesis may be related to perturbations of the complex intracellular and intercellular networks that link tissues and organ systems (20-22). Hence, the pathogenesis of tooth agenesis may be more heterogeneous than was previously considered. We conclude that additional genes are responsible for tooth agenesis, and it is conceivable that a number of these genes also belong to the large group of genes that are known to be expressed during tooth morphogenesis.

The third explanation could be environmental factors. Due to its peculiar properties, during tooth development, variations in the metabolic status of the individual $(23,24)$ may play a role. Our study proved that none of the polymorphisms (c. $956+16 \mathrm{~A}>\mathrm{G}, \mathrm{c} .1365 \mathrm{~A}>\mathrm{G}$ and c. $1200+71 \mathrm{~A}>\mathrm{G}$ ) appear to lie on xenobiotic metabolising genes. At this point, it is essential to take into account the fact that isolated oligodontia is a multifactorial process, and genetic-determined suboptimal xenobiotic metabolising machinery could partly explain not all but certain oligodontia cases, which have developed under certain exposure conditions. The human body is a complex open system. Whether an individual will be affected following contact with harmful factors not only depends on environmental response genes and environmental factors, but also on the complex regulatory processes of various cells, tissues and organs, which cause changes in gene expression. Hence, tooth anomalies may reflect environmental and systemic disturbances. A number of environmental factors $(25,26)$ including irradiation, chemotherapeutic agents and dioxin are capable of arresting tooth development. The dental phenotype could be similar for various genotypes, adverse environments or systemic anomalies, and thus the search for etiological factors should be extremely accurate. These factors may be pivotal in the strategy of molecular screening. Taken together, our results may explain why the polymorphic locus can exhibit the typical edentulous phenotype, and why the same mutation but in heterozygotic form was identified in the proband's mother, and could serve as a starting point for future studies in which pollutant activity and genotype influence could be studied.

Although significant progress has been made in our understanding of the molecular mechanisms underlying oligodontia (27-29), a detailed picture of the genetic background of this syndrome is still lacking. In this study, two individuals in a family affected by the c.956+16A $>\mathrm{G}, \mathrm{c} .1365 \mathrm{~A}>\mathrm{G}$ and c. $1200+71 \mathrm{~A}>\mathrm{G}$ homozygotic mutations lacked permanent teeth, which may indicate that these polymorphisms are a risk factor for oligodontia in the Chinese Han population. We provide further evidence suggesting a role of AXIN2 in tooth agenesis, and also confirm the significance of the Wnt pathway in tooth development. We suggest that other factors, as yet unknown, may be associated with this common developmental anomaly. Although only performed on a few cases, this study on the subject of oligodontia may contribute to our knowledge of the condition. However, to understand the exact function of AXIN2 in odontogenesis requires further detailed analysis of each stage of this process.

\section{Acknowledgements}

The present study was supported by the Municipal Bureau of Science and Technology of Lianyungang city (grant no. SH1332). The authors are grateful to the patients and their family members for their kind cooperation and participation.

\section{References}

1. Matalova E, Fleischmannova J, Sharpe PT and Tucker AS: Tooth agenesis: from molecular genetics to molecular dentistry. J Dent Res 87: 617-623, 2008.

2. Cudney SM and Vieira AR: Molecular factors resulting in tooth agenesis and contemporary approaches for regeneration: a review. Eur Arch Paediatr Dent 13: 297-304, 2012.

3. Lammi L, Arte S, Somer M, et al: Mutations in AXIN2 cause familial tooth agenesis and predispose to colorectal cancer. Am J Hum Genet 74: 1043-1050, 2004.

4. Swinnen S, Bailleul-Forestier I, Arte S, et al: Investigating the etiology of multiple tooth agenesis in three sisters with severe oligodontia. Orthod Craniofac Res 11: 24-31, 2008.

5. Jia S, Zhou J, Gao Y, et al: Roles of Bmp4 during tooth morphogenesis and sequential tooth formation. Development 140: 423-432, 2013 . 
6. Bailleul-Forestier I, Molla M, Verloes A and Berdal A: The genetic basis of inherited anomalies of the teeth: Part 1. Clinical and molecular aspects of non-syndromic dental disorders. Eur J Med Genet 51: 273-291, 2008.

7. Wang J, Jian F, Chen J, et al: Sequence analysis of PAX9, MSX1 and AXIN2 genes in a Chinese oligodontia family. Arch Ora Biol 56: 1027-1034, 2011.

8. Bergendal B, Klar J, Stecksén-Blicks C, et al: Isolated oligodontia associated with mutations in EDARADD, AXIN2, MSX1, and PAX9 genes. Am J Med Genet A 155: 1616-1622, 2011.

9. Gunbay T, Koyuncu BO, Sipahi A, et al: Multidisciplinary approach to a nonsyndromic oligodontia patient using advanced surgical techniques. Int J Periodontics Restorative Dent 31: 297-305, 2011.

10. Nakatomi M, Wang XP, Key D, et al: Genetic interactions between Pax 9 and Msx1 regulate lip development and several stages of tooth morphogenesis. Dev Biol 340: 438-449, 2010.

11. De Coster PJ, Marks LA, Martens LC and Huysseune A: Dental agenesis: genetic and clinical perspectives. J Oral Pathol Med 38 $1-17,2009$

12. Letra A, Menezes R, Granjeiro JM, et al: AXIN2 and CDH1 polymorphisms, tooth agenesis, and oral clefts. Birth Defects Res A Clin Mol Teratol 85: 169-173, 2009.

13. Mostowska A, Biedziak B and Jagodzinski PP: Axis inhibition protein 2 (AXIN2) polymorphisms may be a risk factor for selective tooth agenesis. J Hum Genet 51: 262-266, 2006.

14. Liang J, Song G, Li Q, et al: Novel missense mutations in PAX9 causing oligodontia. Arch Oral Biol 57: 784-789, 2012.

15. Vieira AR: Oral clefts and syndromic forms of tooth agenesis as models for genetics of isolated tooth agenesis. J Dent Res 82 $162-165,2003$.

16. Zhang YD, Chen Z, Song YQ, et al: Making a tooth: growth factors, transcription factors, and stem cells. Cell Res 15: 301-316, 2005.

17. Amen M, Liu X, Vadlamudi U, et al: PITX2 and $\beta$-catenin interactions regulate lef-1 isoform expression. Mol Cel Biol 27: $7560-7573,2007$.
18. Kangas AT, Evans AR, Thesleff I, et al: Nonindependence of mammalian dental characters. Nature 432: 211-214, 2004.

19. Küchler EC, Lips A, Tannure PN, et al: Tooth agenesis association with self-reported family history of cancer. J Dent Res 92: 149-155, 2013.

20. Paixao-Cortes VR, Braga T, Salzano FM, et al: PAX9 and MSX1 transcription factor genes in non-syndromic dental agenesis. Arch Oral Biol 56: 337-344, 2011.

21. Menezes R, Marazita ML, Goldstein McHenry T, et al: AXIS inhibition protein 2 , orofacial clefts and a family history of cancer. J Am Dent Assoc 140: 80-84, 2009.

22. Ruhin-Poncet B, Ghoul-Mazgar S, Hotton D, et al: Msx and dlx homeogene expression in epithelial odontogenic tumors. J Histochem Cytochem 57: 69-78, 2009.

23. Kapadia H, Mues G and D'Souza R: Genes affecting tooth morphogenesis. Orthod Craniofac Res 10: 105-113, 2007.

24. Ovári G, Molnár B, Tarján I, et al: Gene polymorphisms in periodontitis and hypodontia: methodological basis of investigations. Fogorv Sz 100: 266-272, 259-265, 2007.

25. Townsend G, Bockmann M, Hughes T, et al: Genetic, environmental and epigenetic influences on variation in human tooth number, size and shape. Odontology 100: 1-9, 2012.

26. Wang $J$ and Abate-Shen C: Transcriptional repression by the Msx1 homeoprotein is associated with global redistribution of the H3K27me 3 repressive mark to the nuclear periphery. Nucleus 3: 155-161, 2012.

27. Pani SC: The genetic basis of tooth agenesis: basic concepts and genes involved. J Indian Soc Pedod Prev Dent 29: 84-89, 2011.

28. Pinheiro Rdos S, Otero RA, Portela MB and Castro GF: Severe oligodontia and dental anomalies in a child with a history of multiple natal teeth: An eight-year retrospective. Gen Dent 59: e248-e250, 2011.

29. Bural C, Oztas E, Ozturk S, et al: Multidisciplinary treatment of non-syndromic oligodontia. Eur J Dent 6: 218-226, 2012. 